Cómo citar este artículo:

M. G. Vieira-Ferreira, W. A. Dos Santos, J. E. Espíndola-Díaz, "Ground station for miniaturized satellites: a state of the art survey. Revista Ingeniería, Investigación y Desarrollo, vol. 19 (1), pp. 56-63, junio. 2019.

\title{
GROUND STATION FOR MINIATURIZED SATELLITES: A STATE OF ART SURVEY
}

\author{
Estado del arte sobre estaciones terrenas para pequeños satélites \\ Mauricio Gonçalves Ferreira1 ${ }^{1}$, Walter Abrahão dos Santos ${ }^{1}$, Jorge Enrique Espíndola Diaz ${ }^{2}$ \\ ${ }^{1}$ Instituto Nacional de Pesquisas Espaciais- INPE, Engenharia e Tecnologia Espaciais, São José dos Campos, \\ Brasil,Email:mauricio@ccs.inpe.br,walter.abrahao@inpe.br \\ 2Universidad Pedagógica y Tecnológica de Colombia, Escuela de Ingeniería de Sistemas y Computación, \\ Grupo de Investigación INFELCOM, Colombia.Email: jespindola@uptc.edu.co
}

(Recibido Mayo 05 de 2019 y aceptado Agosto 02 de 2019)

\section{Resumen}

Para profundizar en el conocimiento de las estaciones terrenas para el control de pico-satélites y nano-satélites, es necesario conocer el estado del arte de la automatización, la evolución y los últimos avances en esta tecnología. Además, es necesario conocer: protocolos de comunicación utilizados; proyectos de CubeSat que se desarrollaron o se están desarrollando actualmente; organizaciones que regulan el uso de pico-satélites; el diseño de estaciones terrenas; el software más utilizado para rastrear satélites y, por último, investigar las técnicas conocidas para rastrearlos. Este trabajo es una recopilación de los trabajos publicados sobre la evolución del sistema de tierra de los últimos 10 años. Además, proporciona un análisis resumido y crítico sobre el estado del arte de las estaciones terrenas. El objetivo de este trabajo es realizar el estado del arte de las estaciones terrenas para pequeños satélites, evaluar la existencia del uso de técnicas de inteligencia artificial en la automatización de estaciones terrenas e identificar cómo las están trabajando, en instituciones en alrededor del mundo.

Palabras clave: Inteligencia artificial, cubesat, red de estaciones terrestres, pequeños satélites.

\begin{abstract}
In order to deepen the knowledge on ground stations for pico-satellite and nano-satellite control, it is necessary to know the state of the art of automation, the evolution and the latest developments in this technology. Also, it is necessary to know: used communication protocols; CubeSat projects that were developed or are currently being developed; organizations that regulate the use of pico-satellites; the design of ground stations; the most used software for tracking satellites, and to look into known techniques for satellite tracking. This work is a compilation of the papers published concerning the evolution of ground system in the last 10 years. In addition, it provides a summary and critical analysis on state of the art to ground station. The objective of this work is to make a state of the art in ground station for miniaturized satellites, to evaluate the existence of the use of artificial intelligence techniques in the automation of ground stations and identify how institutions are working on ground station around the world. .
\end{abstract}

Key words: Artificial Intelligence, cubesat, ground Station Network, small satellites. 


\section{INTRODUCTION}

The constant evolution of satellite communication platforms demands the use of the modern approaches to system design. Currently in the pipeline, the up-and-coming new wave of smaller satellites is nano-satellites, ranging from 1 to 10 kilogram, and pico-satellite that weighs less than 1 kilogram. The envisioned smallest category is the femto-satellite with less than one-tenth of a kilogram, a satellite that would handle very simple missions and would be implemented on a single chip; this is why they are also called satellites-on-a-chip.

Every satellite, small, medium or large, need to be monitored from Control Centers and Ground System also referred as Ground Station, it was designed for that purpose. These stations must be completely automated or there are largely to guarantee easy access to every kind of user.

Taking into account the development of state of the art automation ground stations, in this article aims to know what has been the evolution of this technology, communication protocols that are used, what kind of small sateIlites are being developed, standards that are used for the creation of a ground station, which the tracking track and control software have been developed, standards that are used for the development of small satellites such as CubeSat, and finally to know if they have used artificial intelligence techniques in the automation of ground stations.

The review of state of the art on ground system was examined and its information organized by the year, the authors, the place and the concept developed. In order to get a better understanding the state of the art on ground system automation. The results and information were classified into four areas: automation of ground station, ground station network, artificial intelligence and ground station virtual. This information is presented afterwards.

\section{METHODOLOGY}

As part of the methodology, the first thing that was done was a compilation of articles published on issues related to the automation of ground systems in the last ten years, indicating the author, year, country, university or research center and contribution in this technology.

After reading these articles, some tables were built with a brief description of each of the studies reviewed. The articles were classified and separated into four areas of interest, making a summary and a critical analysis of the ground stations state of the art.

The information was taken from articles and specialized academic events around the world, articles that were published on indexed journals in the last ten years and articles published on websites.

\section{ANALYSIS OF THE STATE OF THE ART ABOUT AUTO- MATION OF GROUND SYSTEMS}

After reviewing the state of the art on ground system automation, the information found was classified in four areas: automation of ground station, ground station network, use of artificial intelligence and ground station virtual (see figure 1). This is because these issues are part of a ground system and ranking facilitates their study. Although, it describes the information found and an analysis of the advantages, opportunities and strong points, as well as the disadvantages, limitations and weak points found it. GROUND SYSTEM AUTOMATION

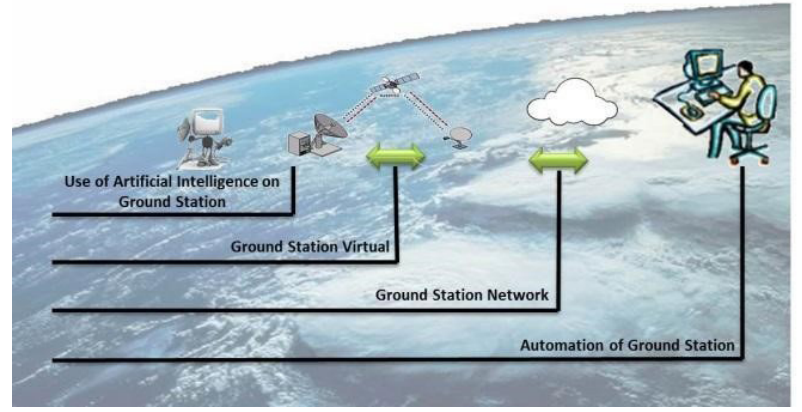

Figure 1. Areas of the ground system.

\subsection{Automation of Ground Stations}

The different tasks and facilities in networks with new features. Keywords for the new ground systems 
are for instance: open systems, automation, "internet" technology, multi-session operations, ground station networks and increasing on-board autonomy [1]. German Aerospace Center DRL, Germany.

This job describes the procedures for automatic tracking of small satellites to receive Beacon signals [2]. Information and Communication Technologies Center. National University of Engineering. Peru.

The system can operate as a real time maneuvering system, executing commands sent from the ground or as a standalone attitude control system receiving the solar array status from a hosting satellite and the satellite ephemeris transmitted from the ground station [3]. Department of Mechanical and Aerospace Engineering. Italy.

This job discusses the automated operations approach being developed for the SMAP GDS [4]. Jet propulsion Laboratory. California Institute of Technology. United States.

The paper also highlights the automation decision taken to reduce the number of operators while managing up to 6 passes per day and per satellite over the command and control antenna for satellites commanding and programming [5]. Astrium Satellites. France.

Providing an overview of the overall monitoring and control concepts for ESTRACK ground stations, highlighting automation aspects for the configuration of the ESTRACK network [6]. ESA. Germany.

\section{Advantages/opportunities/ strong points in Automa- tion of Ground Station}

The analyze shows that works have already automated, such as: monitoring system, attitude control systems, specific project operation, programming of satellite passes on ground stations, as a system of ground stations, for example CELESTRACK of ESA.

It is noted that the ESA is automating network GSMC (Ground Station Monitoring and Control system) Stations.
Therefore, $t$ the space agencies are working on automation of ground system.

An advantage of the automation of ground stations is to reduce considerably the use of qualified personnel, lowering the cost of operation of the station, it means, the automation of the processes of a ground station reduces costs.

\section{Disadvantages/limitations/ weak points in Automation of Ground Station}

The information found about automation of ground stations is neither clear nor accurate. It is not determined how the ground stations have been automated or procedures for tracking satellites.

Based on the amount of items found about this topic, it can be concluded that the ground station automation is still small compared to the amount of ground stations for pico-satellites tracking. Figure 2 shows on a timeline the meaningful work found in this area.

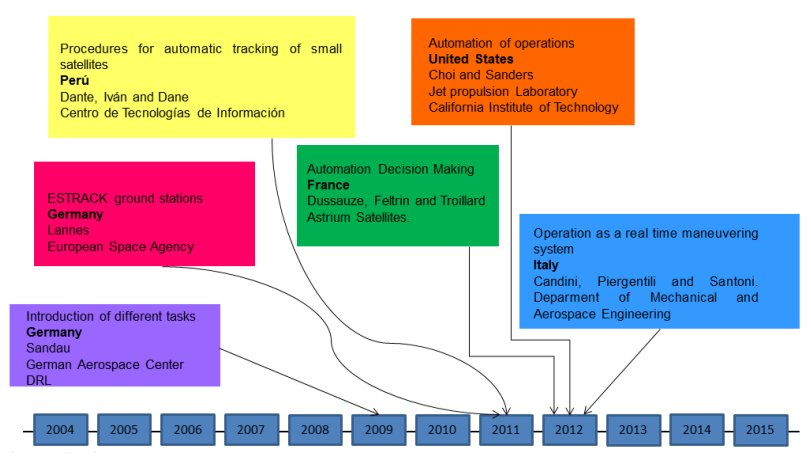

Figure 2. Automation of Ground Stations.

\subsection{Networking Ground Station}

The job focuses on the Configuration Management principles applied during the usage phase in ESA's worldwide network of TT\&C Ground Stations (ESTRACK) [7]. ESA. Germany.

The ESTRACK Planning System (EPS) operationally plans the use of the ESAs ESTRACK ground station network. The system is configured with a specification of the user missions' needs and the networks constituents' capabilities [8]. VEGA, Aerospace Division, European Space Agency. 
Germany.

The ground segment consists of the network of geophysical stations located in several zones of high earthquake and volcanic activity [9]. Institute of Physics of the Earth, Russian Academy of Sciences. Russian Federation.

The support of multiple ground stations in the MCC introduces issues as the use of each ground station must be coordinated and the interfaces to different ground stations are most likely but there are not identical [10]. Department of Control Engineering. Aalborg University. Denmak.

GSN-WG (ground station network working group), with thirteen Japanese universities and four additional participating institutions in the US, Sweden, Taiwan, and Germany [11]. Space Policy Institute, The George Washington University. United States.

Implications of downloaded files and missed opportunities can affect the design of the distributed network of ground stations to support FalconSAT-3 [12]. The Air Force Institute of Technology, Wright-Patterson Air Force Base. United State.

Working with universities around the world to build a loose federation of globally distributed university ground stations [13]. Stanford University, Space System Development Laboratory. United States.

The Distributed Ground Station Network (DGSN) solution can solve the problem with permanent tracking and a faster orbital element provision to the satellite owners, to explore the concept of deploying a network of distributed ground station receiver nodes for the purposes of increasing access time to the spacecraft [14]. University of Stuttgart. Germany.

A master thesis in [15] Virginia Polytechnic Institute, United States explores the concept of deploying a network of distributed ground station receiver nodes for the purposes of increasing access time to the spacecraft.
This job describes the design and operation of the Mercury system, its implementation in the SSDL OSCAR station [16]. Stanford University, Space System Development Laboratory. United States.

Discuss a test bank which consists of globally distribution, university ground station systems. [17]. Stanford University, Space System Development Laboratory. United States.

\section{Advantages/opportunities/ strong points in Networ- king Ground Station}

The ground station networks allows the description of management support tools for ground operations system. It has joined several universities to develop ground stations networks aiming and optimizing the use of resources, eg. GSN- WG.

Using multiple stations connected in a network, improves and monitoring pico-satellites and enables tracking to have a high degree of accuracy. They are creating federations of ground stations distributed around the world to improve the tracking of satellites.

Station networks improves data delivery time to the owners of the satellites, also the access time is increasing the amount of data transmitted. Creating several ground station network as it is evidenced, for instance: SNIP, SSDL, GSN- WG, GSML, etc.

The current trend is the creation of ground stations networks around the world. There is an opportunity to create a ground station network with current stations in Colombia (Universidad Pedagógica y Tecnológica de Colombia, Universidad Distrital, Universidad Sergio Arboleda) with some of Brazil, for example, ground station ITA (Instituto Tecnológico Areonautico), aiming at sharing hardware and software resources.

\section{Disadvantages/limitations/ weak points in Networking Ground Station}

It is important to take some special care with synchronization with the stations that built a network; otherwise 
errors may occur in the quality of the data transmitted.

All stations on the network must have a standard, for example GENSO. The communication protocol must be synchronized for data transmission both the satellite to the station, and between stations. Figure 3 shows what was found about the ground station networks area.

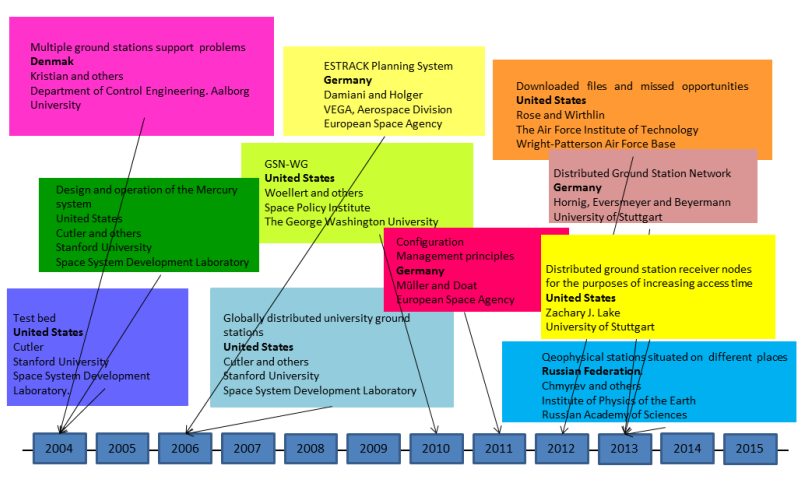

Figure 3. Networking Ground Station.

\subsection{Use of Artificial Intelligence in Ground Station}

In this paper, we present the resolution of the ground station scheduling problem arises in spacecraft operations and aims to allocate ground stations to spacecraft to make possible the communication between operations teams and spacecraft systems through Struggle Genetic Algorithms - a version of GAs that distinguishes for its efficiency in maintaining the diversity of the population during genetic evolution [18]. Seikei University. Japon.

The objective of this job is to provide a survey of neural network trends as applied to the tracking of spacecraft in deep space at Ka-band under several weather conditions, and examine the trade-off between tracking accuracy and communication link performance [19]. Jet Propulsion Laboratory. California Institute of Technology. United States.

This application should automatically control the positioning of the antennas at the station. To design the poisoning control, Artificial Intelligence techniques were used together with selection genetic algorithms, and simulated annealing algorithms [20]. Universidad Pedagógica y Tecnológica de Colombia - UPTC. Colombia.
This schema shows a genetic algorithm optimization for planning satellite ground station antenna layouts. This scheme allows for the optimal placement of large parabolic antennae within a bounded geographic area. International Journal of Electronics, Computer and Communications Technologies, SES Princeton, NJ [21]

\section{Advantages/opportunities/ strong points in Artificial Intelligence}

Using Artificial Intelligence to reduce costs in several management processes of a ground station, improves the management of the same. Artificial intelligence techniques such as neural networks have found solutions to system communications link between the ground system and the satellite.

Automatic positioning of antennas with the use of genetic algorithms allowed the increase and accuracy of tracking the orbit of pico-satellites. There is an opportunity to explore this area of knowledge.

\section{Disadvantages/limitations/ weak points in Artificial In- telligence}

Generally, the Space Agencies do not show how they have used these techniques in their development; this information is limited and classified.

Artificial Intelligence has been used in the development of ground stations. This can be seen through the little of information that was found. Since many times space agencies do not disclose state of the art information on critical areas for consider it as secret, it is possible the existence of more contributions of the artificial intelligence on ground stations than the contributions that were found here. Figure 4 shows the information found in this area.

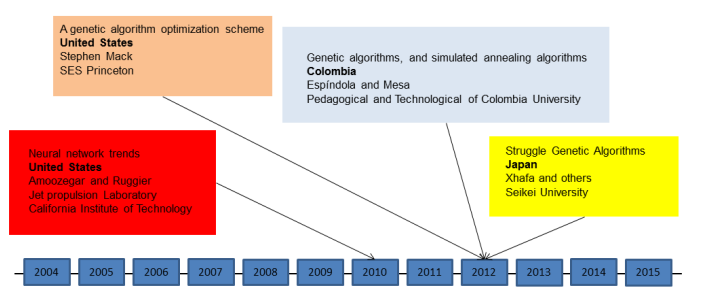

Figure 4. Use of IA on ground stations. 


\subsection{Ground Station Virtualization}

Present the design and implementation of the virtual GS in a higher education and scientific outreach settings [22]. Institute of communication Networks and Satellite Communications/ Space Operations Divisions, Ecuadorian Civilian Space Agency EXA. Austria/Peru.

The Hawaii Space Flight Laboratory (HSFL) in collaboration with NASA, is developing a Comprehensive Open Architecture Space Mission Operations System (COSMOS) to support this and future space missions [23]. Hawaii Space Flight Laboratory, University of Hawaii. United States.

These barriers have reduced by decomposing monolithic ground station functions into basic component services and virtualizing their software and hardware interfaces [24]. Stanford University, Space System Development Laboratory. United Stated.

\section{Advantages/opportunities/ strong points in Virtualiza- tions}

The virtualization ground stations allows more research and science communication on this area of knowledge.

The system virtualization provides tools able to correct errors in real time either, in the past or in the future, improving the performance of the station.

Some universities use virtualization ground station to teach students to solve real problems in this area.

To achieve virtualization ground station is not required large computers; oppositely there is a cheap way to train technical personnel operating the station.

\section{Disadvantages/limitations/ weak points in Virtualiza-} tions

The virtualized system should be understood, it must consider all the variables related to the ground station.

Figure 5 shows the information related to the findings on the virtual ground stations area.

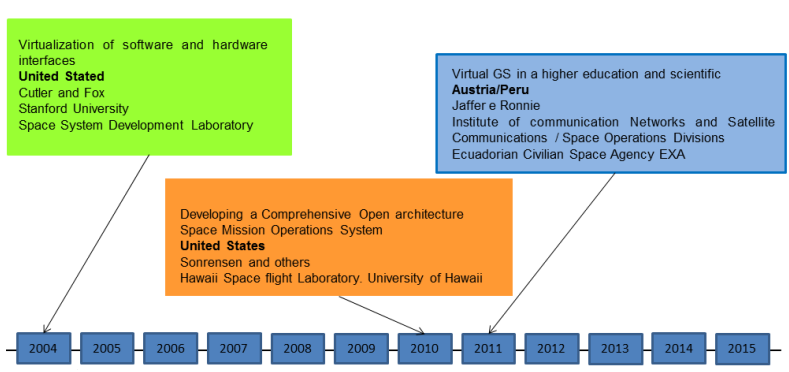

Figure 5. Ground Station Virtualization

\section{CONCLUSIONS}

After the making of the state of the art about ground system for pico-satellites, these conclusions are considered:

The state of art on ground systems for pico-satellites allowed discover four areas of work: automation of ground station, ground station network, artificial intelligence and virtual ground station. This classification facilitated the study and understandings of this document.

The state of the art about ground systems can be deduced that the ground station automation for pico-satellites reduces operating costs and increases the performance of the same time.

Although, Space Agencies have developed ground station automation, it can be concluded that information is not disclosed in a clear and precise way, besides it is considered classified information.

According to the state of art made, the use of artificial intelligence techniques in the ground system development is not significant; however, projects that have used some technique of artificial intelligence have been successful.

Projects such as GSML, SSDL, DGSN, GSMC and others, allow deducting that the current trend is to create ground station networks for pico-satellites management, tracking, monitoring and control. These networks will be distributed throughout the Earth and will have greater importance due to the amount of pico 
and nano-satellites placed into orbit in recent times.

The virtualization of ground stations leads to lower costs, allowing the training of operators and students of the universities.

\section{REFERENCIAS}

[1] Sandau, R., 2009, "Status and trends of small satellite missions for earth observation", Acta Astronautica, Vol.66,pp.1-12.doi:10,1016/j.actaastro.209.06.008.

[2] Dante, I., Velasquez, I., Dane, C. 2011. "Implementation of a Small-Satellites Automatic Tracking System to Reception and Interpretation of Beacon Signals from CTIC-UNI's Ground Station". Revista ECIPERU, Vol. 8 No. 2. Centro de Tecnologías de Información y Comunicaciones. Universidad Nacional de Ingeniería.

[3] Candini, G. P., Piergentili, F., Santoni, F., 2012, “Miniaturized attitude control system for nanosateIlites", Acta Astronautica, Vol. 81, pp. 325-334. doi:10.1016/j.actaastro.2012.07.027.

[4] Choi, J. S., Sanders, A. L., 2012, "Cost-Effective telemetry and command ground systems automation strategy for the soil moisture active passive (SMAP) mission", Spaceops 2012 Conference. doi:10.2514/6.2012-1275978.

[5] Dussauze, J., Feltrin, G., Troillard, J., 2012, "AstroTerra control ground segment: operations concept and implementation", Spaceops 2012 Conference. doi:10.2514/6.2012-1289133.

[6] Lannes, C., Pissias, P., Riccio F., 2011, "A new generation of monitoring and control system for ESTRACK", Aerospace 2012 Conference, Vol. 3, pp. 1890-1897 doi: 10.2514/6.2012-1286386.

[7] Müller, P., Doat, Y. 2012, "Configuration management in a ground station network", Spaceops 2012 Conference. doi/abs/10.2514/6.2012-1263858

[8] Damiani, S., Dreihahn H., Noll, J., Niezette, M., Calzolari, G., 2006, "Automated allocation of ESA ground station network services", American Association for Artificial Intelligence, D-64293, Darmstadt, Germany.
[9] Chmyrev, V., Smith A., Kataria D., Nesterov B., Owen C., Sammonds P., Sorokin V., Vallianatos F., 2013, "Detection and monitoring of earthquake precursors: TwinSat, a Russia-UK satellite project", Advances in Space Research, Vol. 52, pp. 1135-1145. doi:10.1016/j.asr.2013.06.017.

[10] Edlund, K., Green, M., Kragelund, M., Michelsen, A., Stougaard, R., 2004, "Generic distributed mission control center for student satellites 2004", IEEE Aerospace Conference, Vol. 22.

[11] Woellert, K., Ehrenfreund, P., Ricco, A., Hertzfeld, H., 2010, "Cubesats: cost-effective science and technology platforms for emerging and developing nations", Advances in Space Research, Vol. 47, pp. 663-684. doi:10.1016/j.asr.2010.10.009.

[12] Rose, C. W., Wirthlin, J. R., 2012, "Using m\&s to maximize space satellite data collection with multiple ground stations", Procedia Computer Science, Vol. 8, pp. 124-128. doi:10.1016/jprocs.2012.01.024.

[13] Cutler, J. W., 2004 "Ground station markup language”, 2004 IEEE Aerospace Conference Proceedings, Vol. 21, pp. 3337-3343. NASA grant NAG3-2579 IEEEAC 1561.

[14] Hornig, A.; Eversmeyer, T.; Beyermann, U., 2013. "Distributed ground station network - a global system for tracking and Communication with small satellites as an open service", 64th International Astronautical Congress, Beijing, China, v. 11.

[15] Zachary J. Leffke, 2013. "Distributed ground station network for CubeSat communications". Thesis submitted to the Faculty of the Virginia Polytechnic Institute.

[16] Cutler, J. W., Kitts, C., 2004, "Mercury: a satellite ground station control system", Aerospace conference 1999, Vol. 2 pp. 51-58. doi:10.1109/ AERO.1999.793142

[17] Cutler, J. W., Fox, A., 2006, "A framework for robust and flexible ground station networks", Journal of Aerospace Computing, Information and Communication, Vol. 3, pp. 73-92. doi:10.2514/1.15464

[18] Xhafa, F., Herrero, X., Barolli, A., Barolli, L., Takizawa, M., 2013 "Evaluation of struggle strategy. 
in genetic algorithms for ground stations scheduling problem", Journal of Computer and System Sciences, Vol. 79, pp. 1086-1100. doi:10.1016/j. jcss.2013.01.023.

[19] Amoozegar, F., Ruggier, C., 2010, "Neural network based satellite tracking for deep space applications", Automatic Target Recognition XIII, pp. 119-135. SPIE 0277-786X/03

[20] Espindola, J. E.; Mesa, J.A., 2012, "Automatic addressing scheme for antennas at pico-satellite tracking ground station", Revista Tecnura, Vol. 17, No. 35, pp. 26-37. from http://tecnura.udistrital.edu.co/ ojs/index.php/revista/rt/printerFriendly/513/601

[21] Mack S. 2012. "Optimization of a Satellite Earth Station Layout Using Genetic Algorithms". International Journal of Electronics, Computer and Communications Technologies, SES Princeton, NJ.

[22] Jaffer, G., Nader, R., Koudelka, O., 2011, "Internet-to-orbit gateway and virtual ground station: a tool for space research and scientific outreach", Acta Astronautica, Vol. 69, pp. 658-666. doi:10.1016/j. actaastro.201.05.021.

[23] Sorensen, T., Pilguer, E., Wood, M., Gregory, E., Nunes, M., 2010, "Development of the mission operations support tool (MOST)", SpaceOps 2010 Conference, doi:10.2514/6.2010-2230.

[24] Cutler, J. W., 2004, "Ground Station Virtualization", in Proceedings of the Fifth International Symposium on Reducing the Cost of Spacecraft Ground Systems and Operations (RCSGSO), Pasadena, CA. 